\title{
CASE REVIEW
}

\section{A link between asthenia, pallor, and jaundice}

\author{
João Tavares physician in general internal medicine ${ }^{1}$, Gisela Eugénio resident in rheumatology ${ }^{2}$, \\ Suzana Calretas consultant physician in general internal medicine ${ }^{3}$, Rui Silva pathologist ${ }^{4}$, Rui \\ Santos consultant physician in general internal medicine and clinical professor of medicine ${ }^{3}$, Armando \\ Carvalho consultant physician in general internal medicine and clinical professor of medicine ${ }^{3}$
}

\footnotetext{
${ }^{1}$ Hospitais da Universidade de Coimbra, Coimbra 3000-075, Portugal; '2Department of Rheumatology, Hospitais da Universidade de Coimbra; ${ }^{3}$ Department of Internal Medicine, Hospitais da Universidade de Coimbra; ${ }^{4}$ Department of Pathology, Hospitais da Universidade de Coimbra
}

\begin{abstract}
A 45 year old white man presented to the emergency department with progressively worsening weakness and chest discomfort on moderate intensity physical activity over the past two months. He also reported paleness and "yellowish eyes" in the past month. He had a history of anxiety, treated with alprazolam as needed.
\end{abstract}

At presentation, his vital signs were normal. On physical examination he had mild generalised pallor, scleral icterus, and tender non-painful hepatomegaly.

He was admitted to the internal medicine ward for further evaluation.

Laboratory studies showed haemoglobin $70 \mathrm{~g} / \mathrm{L}$ (reference range 13-17), mean corpuscular volume $111.5 \mathrm{fL}$ (83-101), haematocrit $19.4 \%$ (40-50\%), leucocyte count $3.3 \times 10^{9} / \mathrm{L}$ (4-10), platelet count $151 \times 10^{9} / \mathrm{L}(150-450)$, reticulocyte production index 0.52 $(>2)$, lactate dehydrogenase $1460 \mathrm{U} / \mathrm{L}$ (125-220), total bilirubin $35.92 \mu \mathrm{mol} / \mathrm{L}$ (5.13-20.52), direct bilirubin $11.97 \mu \mathrm{mol} / \mathrm{L}$ (1.71-8.55), haptoglobin $<0.07 \mathrm{~g} / \mathrm{L}(0.30-2)$, vitamin $\mathrm{B}_{12} 57.55$ $\mathrm{pmol} / \mathrm{L}$ (156-672), ferritin $177 \mathrm{ng} / \mathrm{mL}$ (50-250), and folic acid $26.74 \mathrm{nmol} / \mathrm{L}(>12.24)$. The peripheral blood smear showed macrocytosis and hypersegmented neutrophils.

Antibodies against parietal cells were positive; Coombs test and anti-intrinsic factor antibodies were negative.

Upper gastrointestinal endoscopy showed atrophic gastritis of the body and fundus. Staining for Helicobacter pylori was negative (fig 1). Biopsy confirmed the presence of type A atrophic gastritis with moderate intestinal metaplasia.
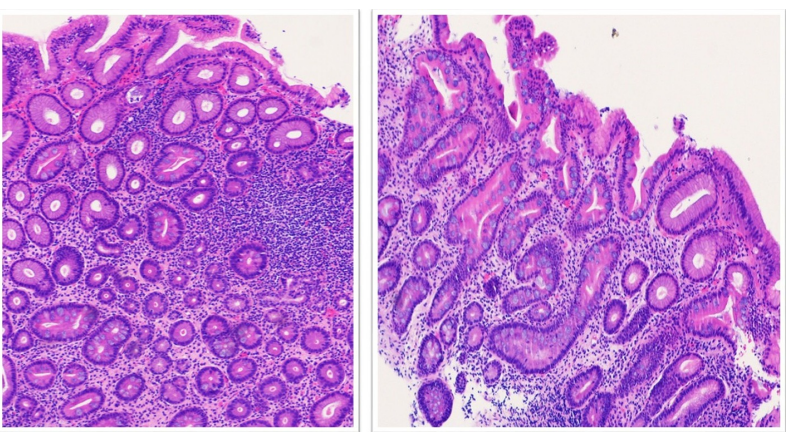

Fig 1 Gastric body fragments showing glandular atrophy plus moderate intestinal metaplasia and a moderate mononuclear infiltrate of the lamina propria (haematoxylin and eosin stain, original magnification $\times 100$ )

\section{Questions}

1.What is the most likely diagnosis?

2.What strategy can primary care practitioners take to reach the diagnosis?

3.What are the differential diagnoses?

4.How is this condition managed?

5.How is this condition followed up?

\section{Answers}

1.

\section{What is the most likely diagnosis?} Short answer

Pernicious anaemia with intramedullary haemolysis as a result of ineffective erythropoiesis. 


\section{Discussion}

Pernicious anaemia is a macrocytic anaemia caused by vitamin $\mathrm{B}_{12}$ (cobalamin) deficiency, as a consequence of an intrinsic factor deficit. The deficiency in intrinsic factor is caused by an autoimmune disease that targets gastric parietal cells and leads to gastric atrophy of the body and fundus (type A chronic atrophic gastritis). Intrinsic factor aids the absorption of vitamin $\mathrm{B}_{12}$ in the terminal ileum, so its deficiency results in low levels of vitamin $\mathrm{B}_{12}$ and in pernicious anaemia. ${ }^{12}$

The prevalence of pernicious anaemia ranges from 50 to 4000 cases per 100000 people. $^{3}$ The mean age of diagnosis is 50-60 years. ${ }^{2}$ Its presentation is highly variable, ranging from asymptomatic disease in the early stages to symptoms of anaemia, such as dyspnoea on exertion, palpitations, and asthenia. It sometimes presents with neurological manifestations, from peripheral neuropathy (commonly paraesthesia and numbness) to signs and symptoms of spinal cord injury (loss of vibration and position sense with sensory ataxia and limb weakness). Cerebral manifestations such as delusions, hallucinations, and paranoid schizophrenic ideation ("megaloblastic madness") can also be present. ${ }^{34}$

Haemolysis can also occur as a result of DNA synthesis and nuclear maturation defects, which lead to ineffective erythropoiesis and intramedullary haemolysis, ${ }^{3}$ and autoimmune thyroid disease occurs in $18-40 \%$ of patients.

Even though pernicious anaemia is considered a benign disease, patients are prone to the development of intestinal-type gastric adenocarcinoma and gastric carcinoid type I. The incidence rate of gastric cancer is $0.27 \%$ per person years-a seven times higher relative risk than in the general population. ${ }^{5}$ The main reasons for this increased risk are the presence of hypochlorhydria or achlorhydria, which leads to hypergastrinaemia (risk factor for enterochromaffin-like cell hyperplasia and gastric carcinoids) and overgrowth of carcinogenic nitrosamine producing bacteria, which also contributes to the development of gastric cancer. ${ }^{56}$

\section{2.}

\section{What strategy can primary care practitioners take to reach the diagnosis?}

\section{Short answer}

Request tests for hypoproliferative macrocytic anaemia and serum cobalamin levels. ${ }^{2-8}$ Request endoscopic examination with histological analysis. ${ }^{68}$

\section{Discussion}

There are no defined criteria for the diagnosis of pernicious anaemia because it can present with a variety of laboratory, endoscopic, and histological findings (box 1). However, it is accepted that the demonstration of megaloblastic anaemia, macro-ovalocytes, and hypersegmented neutrophils in the blood smear; low serum vitamin $B_{12}$ levels; atrophic gastritis (body or fundus); and the presence of antibodies to gastric parietal cells or intrinsic factor are sufficient to make the diagnosis. ${ }^{2}$

Diagnosis is based on the presence of macrocytic anaemia with a low reticulocyte production index, reduced serum cobalamin levels, and the presence of atrophic body gastritis and intrinsic factor deficiency. ${ }^{8}$

Importantly, the measurement of serum cobalamin can be misleading, especially for values around the lower limit of normal. ${ }^{3}$ In this case, the diagnosis should be confirmed by determining plasma methylmalonic acid and total homocysteine, which are increased in the presence of a cobalamin deficit.
Holotranscobalamin (transcobalamin bound to cobalamin), also referred to as active vitamin $B_{12}$, is considered an early marker of vitamin $B_{12}$ deficiency, with some studies reporting a higher specificity and sensibility than for total vitamin $B_{12}$.

A holotranscobalamin value of $<35-40 \mathrm{pmol} / \mathrm{L}$ is consistent with vitamin $\mathrm{B}_{12}$ deficiency, ${ }^{8}$ although there is no consensus on the optimal cut-off values. The British Society for Haematology suggests using cut-off values that are based on manufacturers' reference ranges or determined by the laboratory performing the test. ${ }^{9}$

Holotranscobalamin can be used instead of total vitamin $B_{12}$ in certain populations, such as pregnant women, because unlike total serum cobalamin it does not show a physiological reduction during pregnancy. ${ }^{9}$

Although holotranscobalamin performs better than total vitamin $\mathrm{B}_{12}$, a confirmatory test (methylmalonic acid or homocysteine) is still needed when results are indeterminate. ${ }^{9-12}$

Once vitamin $B_{12}$ deficiency has been confirmed the cause needs to be determined. Pernicious anaemia is caused by an immune mediated destruction of the gastric parietal cells, which results in achlorhydria with hypergastrinaemia and low levels of intrinsic factor secretion. Thus, the presence of endoscopic signs of chronic atrophic type A gastritis and its histological corroboration are usually considered sufficient to confirm the diagnosis. $^{67}$

Serological positivity to intrinsic factor or parietal cell autoantibodies (or both) supports a diagnosis of pernicious anaemia. Intrinsic factor antibodies have a sensitivity and specificity of $37 \%$ and $100 \%$, respectively (enzyme linked immunosorbent assay; ELISA), whereas parietal cell antibodies have a sensitivity and specificity of $81.5 \%$ and $90.3 \%$, respectively. ${ }^{2}$ Although parietal cell antibodies have the higher sensitivity, they can be absent in the later stages of the disease because of a reduction in the number of target (parietal) cells. ${ }^{6}$

A proposed general approach to the diagnosis of pernicious anaemia is shown in fig 2 .

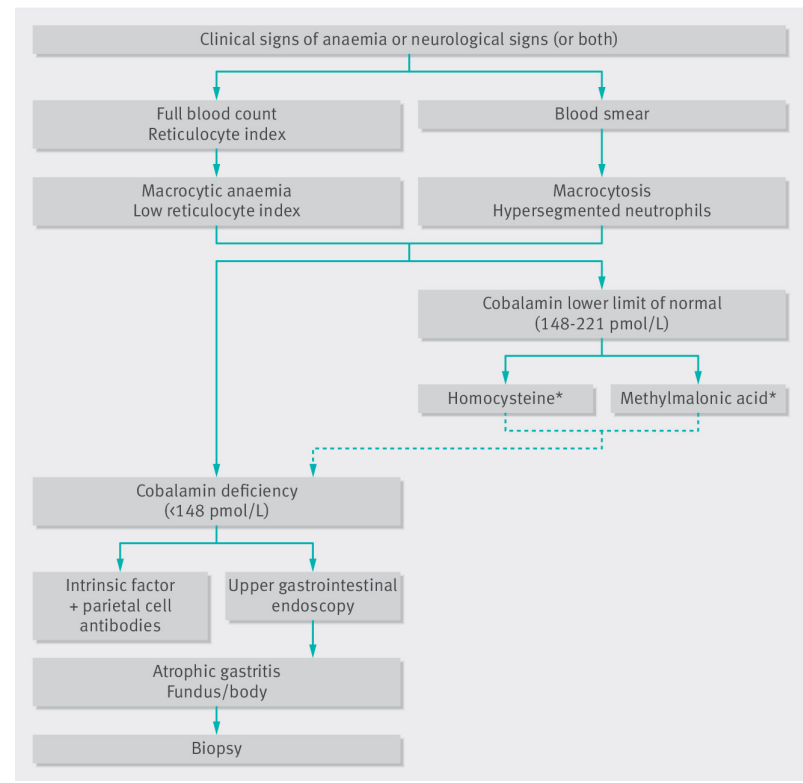

Fig 2 Diagnostic approach to pernicious anaemia. *High levels suggest cobalamin deficiency

Iron studies should also be considered in the initial assessment of pernicious anaemia because iron absorption can be impaired when gastric $\mathrm{pH}$ is raised. ${ }^{13}$ 


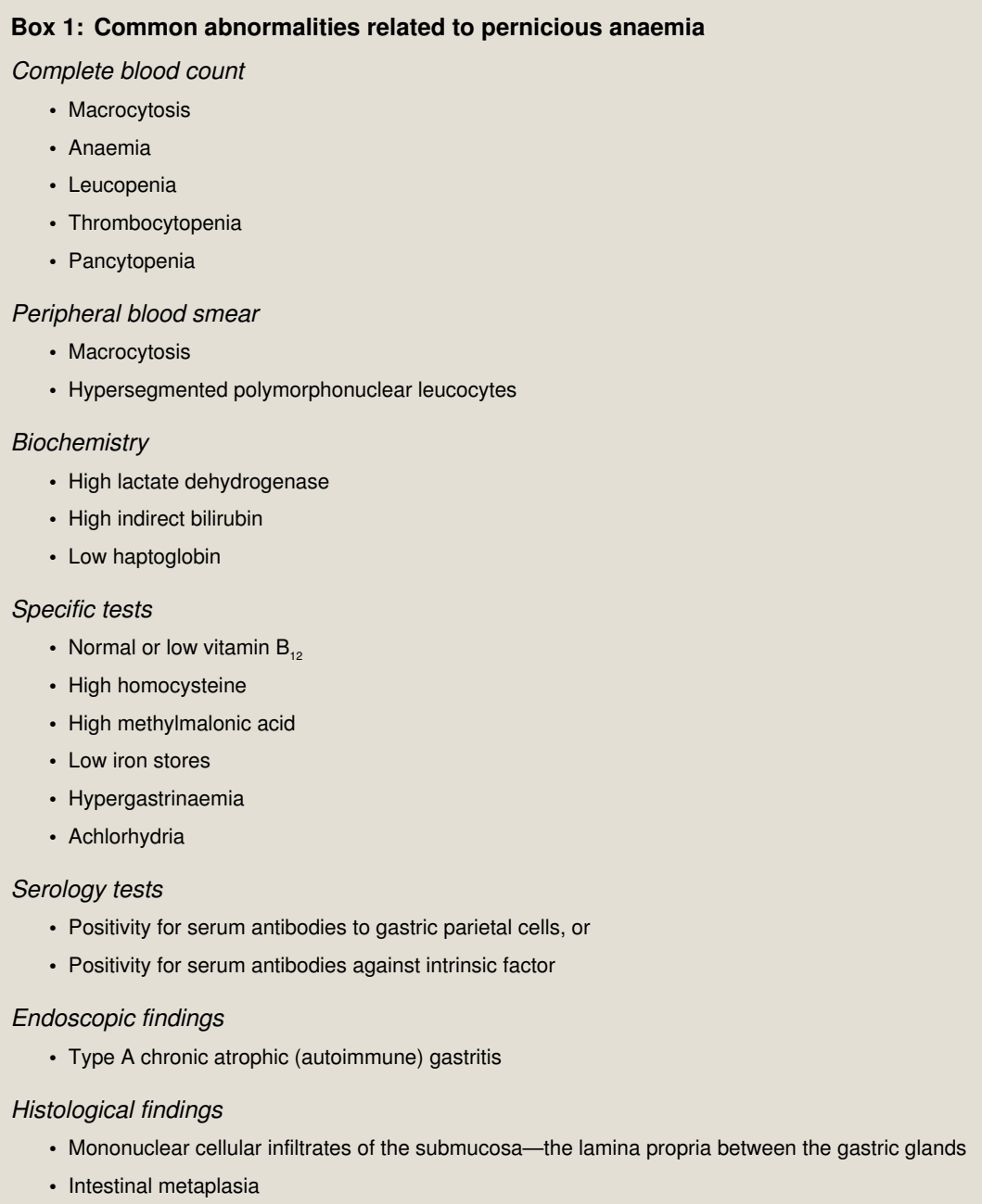

3.

\section{What are the differential diagnoses? Short answer}

Other causes of vitamin $B_{12}$ deficiency, macrocytic anaemia, and intravascular haemolysis.

\section{Discussion}

The differential diagnoses of pernicious anaemia are the same as those of macrocytic anaemia. They include folate deficiency, myelodysplasia, alcoholic liver disease, hypothyroidism, drugs that block folate metabolism (eg methotrexate), and other causes of vitamin $B_{12}$ deficiency (eg partial or total gastrectomy, fish tapeworm infection, Crohn's disease, ileal resection, and a vegan diet) ${ }^{4-15}$ Box 2 lists the common causes of macrocytosis. ${ }^{9-18}$

\section{4. \\ How is this condition managed? Short answer}

With lifelong cyanocobalamin or hydroxocobalamin supplementation. $^{2}$

\section{Discussion}

Pernicious anaemia is treated with cyanocobalamin or hydroxocobalamin. The route, dosage, and schedule of administration vary widely between healthcare institutions and doctors. Box 3 summarises some of the accepted treatment regimens. ${ }^{2-22}$
Lifelong vitamin $B_{12}$ supplementation is warranted. Effective treatment corrects blood counts in 8-12 weeks and improves neurological signs and symptoms within three months if given soon after onset. ${ }^{21} 22$

This condition can be managed by GPs in the community, but referral to specialists should be considered in certain scenarios (box 4).

Supportive therapy with iron and folic acid should be started in patients with severe anaemia to enhance haematopoiesis.

If iron deficiency is present, supplementation should be started promptly. Oral iron formulations are considered first line therapy, with intravenous iron replacement being indicated for patients who do not respond to oral treatment. ${ }^{23}$

Folic acid supplementation can worsen or precipitate neurological manifestations of vitamin $B_{12}$ deficiency. We therefore suggest starting folic acid after at least one dose of vitamin $B_{12}$ and only when folate levels are low or at the lower limit of normal. We also suggest assessing folic acid levels as a part of the initial evaluation and seven days (reticulocytosis peak) after the start of vitamin $B_{12}$ supplementation. Treatment with folic acid should be maintained until anaemia resolves.

\section{5.}

\section{How should this condition be followed up?} Short answer

Annual clinical review, full blood count, thyroid stimulating hormone and free thyroxine assays, serum cobalamin and ferritin analysis, and regular endoscopy for gastric cancer are advised. 


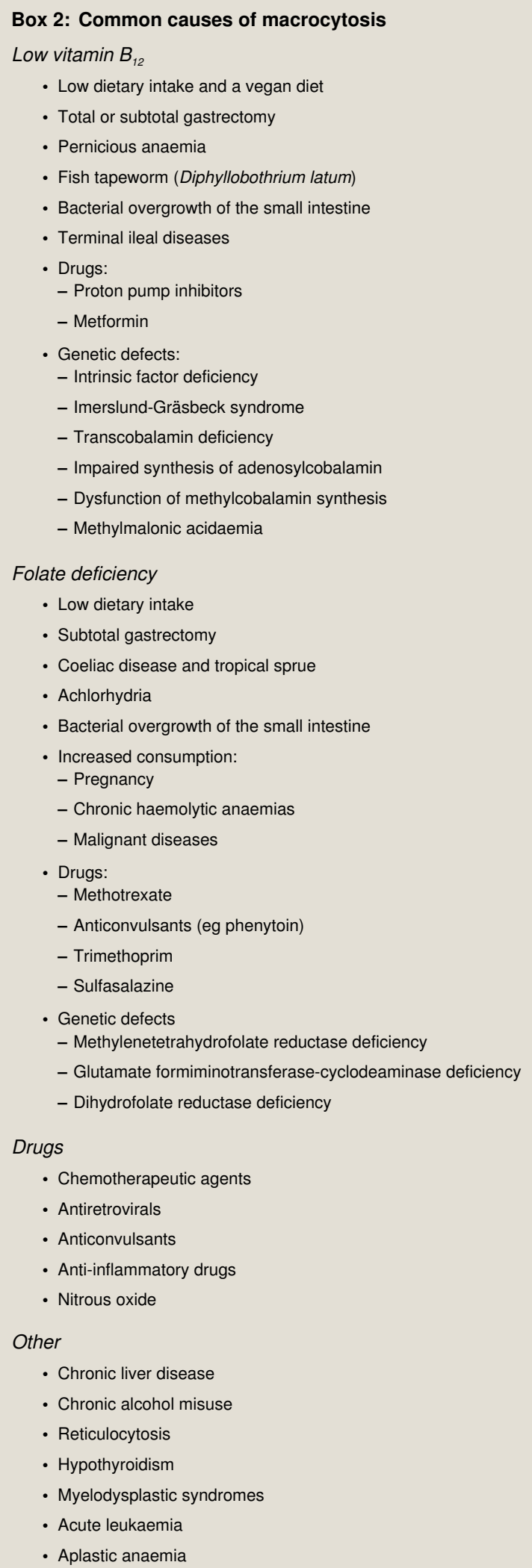

- Chronic alcohol misuse

- Reticulocytosis

- Hypothyroidism

- Myelodysplastic syndromes

- Acute leukaemia

- Aplastic anaemia

GPs can arrange this, with referral to a specialist if associated diseases (autoimmune disease or gastric cancer) are present or if treatment response is inadequate.

\section{Discussion}

Primary care physicians play a crucial role because pernicious anaemia requires lifelong surveillance to ensure the administration of adequate quantities of cobalamin, to prevent the onset of iron deficiency, and to arrange regular endoscopic surveillance for gastric cancer. Furthermore, regular evaluation for autoimmune thyroid disease is warranted. ${ }^{2}{ }^{13}$

The optimal screening strategy for long term complications has not been agreed. Some authors suggest yearly clinical review and laboratory testing (complete blood count, serum cobalamin and ferritin measurement) plus: 


\title{
Box 3: Accepted treatment strategies for pernicious anaemia
}

$1000 \mu \mathrm{g}$ of intramuscular cyanocobalamin given daily for 7-10 days, weekly for four weeks, and then monthly for life ${ }^{2}$

Patients without neurological symptoms ${ }^{9}$

$1000 \mu \mathrm{g}$ of intramuscular hydroxocobalamin given three times a week for two weeks, then $1000 \mu \mathrm{g}$ once every three months for life

Patients with neurological symptoms ${ }^{9}$

$1000 \mu \mathrm{g}$ of intramuscular hydroxocobalamin every two days until no further improvement, followed by $1000 \mu \mathrm{g}$ once every two months for life

$1000-2000 \mu \mathrm{g}$ of oral cyanocobalamin daily ${ }^{*}+$ for life ${ }^{19}$

*Should not be used in patients with symptoms caused by anaemia (haemoglobin $<80 \mathrm{~g} / \mathrm{L}$ ), those with neurological manifestations, or those in hospital.

†Not widely used (except in Sweden and Canada) because most studies supporting this approach show some design or methodological limitations, and vitamin $B_{12}$ has an unreliable absorption rate..$^{190}$

\author{
Box 4: Indications at presentation for referral to specialised care \\ - Consultation with a haematologist or neurologist is recommended if the diagnosis is unclear (eg pancytopenia or neurological \\ manifestations) \\ - Refer to a gastroenterologist if the patient also had iron or folic acid deficiency (or both) to rule out other conditions that can cause \\ malabsorption (eg coeliac disease) and investigate the need for intravenous iron treatment \\ - Consider admission to the emergency department for all patients with severe symptoms of anaemia
}

- Five year follow-up with endoscopy of the upper gastrointestinal tract in patients with enterochromaffin-like cells hyperplasia, or

- Three year follow-up with endoscopy of the upper gastrointestinal tract in patients with pernicious anaemia who are under 60 years, or

- Four year follow-up every four years after diagnosis of pernicious anaemia. $^{2}$

Follow-up endoscopies can be arranged by GPs. If extensive metaplastic atrophy, gastric epithelial dysplasia, adenomas, or dysplasia in hyperplastic polyps is found at endoscopic examination, patients should be referred to gastroenterology for further evaluation. ${ }^{24}$

Patients with certain clinical and laboratory findings during follow-up should be referred to specialised care (box 5).

\section{Patient outcome}

Blood transfusion relieved this patient's symptoms. Vitamin $\mathrm{B}_{12}$ levels were restored with daily $1000 \mu \mathrm{g}$ of cyanocobalamin, administered intramuscularly for seven days, then weekly for four weeks, and monthly thereafter. Supportive treatment with $357 \mathrm{mg}$ of iron(III)-hydroxide polymaltose complex and $5 \mathrm{mg}$ of folic acid, orally once a day, was also started.

After the first week of treatment, his reticulocyte production index rose to $>2$. This confirmed a good treatment response and he was discharged. At follow-up with an internal medicine specialist six weeks later there was an overall improvement, with haemoglobin of $101 \mathrm{~g} / \mathrm{L}$, mean corpuscular volume 102 $\mathrm{fL}$, ferritin $251 \mathrm{ng} / \mathrm{mL}$, and cobalamin $2000 \mathrm{pg} / \mathrm{mL}$.

During the first year of follow-up he will be evaluated by internal medicine and will then be followed up by his GP. Clinical evaluation, a full blood count, ferritin, and vitamin $B_{12}$ assessment are warranted, as repeat endoscopy of the upper gastrointestinal tract in the next three years.

Competing interests: We have read and understood BMJ policy on declarations of interest and declare that we have none.

Provenance and peer review: Commissioned; not externally peer reviewed.

Patient consent obtained.
1 García-Carrasco M, Jiménez-Hernández M, Mendoza-Pinto C, Ruiz-Argüelles A, Fuentes-Alexandro S. Pernicious anemia. In: Shoenfeld Y, Cervera R, Gershwin ME, eds. Diagnostic criteria in autoimmune diseases. Humana Press, 2008:513-7. doi:10.1007/978-1-60327-285-8_94. 2 Annibale B, Lahner E, Fave GD. Diagnosis and management of pernicious anemia. Curr Gastroenterol Rep 2011;13:518-24. doi:10.1007/s11894-011-0225-5 pmid:21947876.

3 Stabler SP. Clinical practice. Vitamin B12 deficiency. N Engl J Med 2013;368:149-60. doi:10.1056/NEJMcp1113996 pmid:23301732.

4 Okam MM. Anemia and hemoglobinopathies. In: The Brigham intensive review of internal medicine. 2nd ed. Oxford University Press, 2014: 185-97.

5 Vannella L, Lahner E, Osborn J, Annibale B. Systematic review: gastric cancer incidence in pernicious anaemia. Aliment Pharmacol Ther 2013;37:375-82. doi:10.1111/apt. 12177 pmid:23216458.

6 Toh BH, van Driel IR, Gleeson PA. Pernicious anemia. N Engl J Med 1997;337:1441-8. doi:10.1056/NEJM199711133372007 pmid:9358143.

7 Cattan D. Pernicious anemia: what are the actual diagnosis criteria? World J Gastroenterol 2011;17:543-4. doi:10.3748/wjg.v17.i4.543 pmid:21274387.

8 Lahner E, Annibale B. Pernicious anemia: new insights from a gastroenterological point of view. World J Gastroenterol 2009;15:5121-8. doi:10.3748/wjg.15.5121 pmid:19891010.

9 Devalia V, Hamilton MS, Molloy AM. British Committee for Standards in Haematology. Guidelines for the diagnosis and treatment of cobalamin and folate disorders. Br J Haematol 2014;166:496-513. doi:10.1111/bjh.12959 pmid:24942828.

10 Herrmann W, Obeid R, Schorr H, Geisel J. The usefulness of holotranscobalamin in predicting vitamin B12 status in different clinical settings. Curr Drug Metab 2005;6:47-53. doi:10.2174/1389200052997384 pmid:15720207.

11 Nexo E, Hoffmann-Lücke E. Holotranscobalamin, a marker of vitamin B-12 status: analytical aspects and clinical utility. Am J Clin Nutr 2011;94:359S-65S. doi:10.3945/ajcn.111. 013458 pmid:21593496.

12 Miller JW, Garrod MG, Rockwood AL, et al. Measurement of total vitamin B12 and holotranscobalamin, singly and in combination, in screening for metabolic vitamin B12 deficiency. Clin Chem 2006;52:278-85. doi:10.1373/clinchem.2005.061382 pmid:16384886.

13 Hershko C, Camaschella C. How I treat unexplained refractory iron deficiency anemia. Blood 2014;123:326-33. doi:10.1182/blood-2013-10-512624 pmid:24215034.

14 Aslinia F, Mazza JJ, Yale SH. Megaloblastic anemia and other causes of macrocytosis. Clin Med Res 2006;4:236-41. doi:10.3121/cmr.4.3.236 pmid:16988104.

15 Puig A, Mino-Kenudson M, Dighe AS. Case records of the Massachusetts General Hospital. Case 13-2012. A 62-year-old man with paresthesias, weight loss, jaundice, and anemia N Engl J Med 2012;366:1626-33. doi:10.1056/NEJMcpc1111575 pmid:22533579.

16 Wickramasinghe SN. Diagnosis of megaloblastic anaemias. Blood Rev 2006;20:299-318. doi:10.1016/j.blre.2006.02.002 pmid:16716475.

17 Kaferle J, Strzoda CE. Evaluation of macrocytosis. Am Fam Physician 2009;79:203-8.pmid: 19202968.

18 Whitehead VM. Acquired and inherited disorders of cobalamin and folate in children. $\mathrm{Br}$ $J$ Haematol 2006;134:125-36. doi:10.1111/j.1365-2141.2006.06133.x pmid:16846473.

19 Vidal-Alaball J, Butler CC, Cannings-John R, et al. Oral vitamin B12 versus intramuscular vitamin B12 for vitamin B12 deficiency. Cochrane Database Syst Rev 2005;3:CD004655.pmid:16034940

20 Sanz-Cuesta T, González-Escobar P, Riesgo-Fuertes R, et al. OB12 Group. Oral versus intramuscular administration of vitamin B12 for the treatment of patients with vitamin B12 deficiency: a pragmatic, randomised, multicentre, non-inferiority clinical trial undertaken in the primary healthcare setting (Project OB12). BMC Public Health 2012;12:394. doi: 10.1186/1471-2458-12-394 pmid:22650964.

21 Dali-Youcef N, Andrès E. An update on cobalamin deficiency in adults. QJM 2009;102:17-28. doi:10.1093/qjmed/hcn138 pmid:18990719.

22 Carmel R. How I treat cobalamin (vitamin B12) deficiency. Blood 2008;112:2214-21. doi: 10.1182/blood-2008-03-040253 pmid:18606874.

23 Bayraktar UD, Bayraktar S. Treatment of iron deficiency anemia associated with gastrointestinal tract diseases. World J Gastroenterol 2010;16:2720-5. doi:10.3748/wjg. v16.i22.2720 pmid:20533591.

24 Neumann WL, Coss E, Rugge M, Genta RM. Autoimmune atrophic gastritis-pathogenesis, pathology and management. Nat Rev Gastroenterol Hepatol 2013;10:529-41. doi:10. 1038/nrgastro.2013.101 pmid:23774773. 
Box 5: Referral for specialist evaluation during follow-up and increased risks associated with pernicious anaemia

- Changes in symptoms or laboratory findings suggestive of haematological, upper airway, or digestive system cancers should prompt referral to specialised care

- The risk of haematological, tonsillar, hypopharyngeal, gastric, small intestine, and oesophageal cancers is increased in elderly people with pernicious anaemia ${ }^{25}$

- Referral to an internal medicine specialist and other specialists (eg endocrinologist) should be considered if concomitant autoimmune diseases are suspected

- Associations between pernicious anaemia and autoimmune thyroid disease, type 1 diabetes, and Addison's disease have been described $^{132}$

25 Murphy G, Dawsey SM, Engels EA, et al. Cancer risk after pernicious anemia in the US elderly population. Clin Gastroenterol Hepatol 2015;13:2282-9.e4. doi:10.1016/..cgh.2015. 05.040 pmid:26079040.
Published by the BMJ Publishing Group Limited. For permission to use (where not already granted under a licence) please go to http://group.bmj.com/group/rights-licensing/ permissions 